\title{
Reversible Horner's syndrome after cervical spinal cord stimulator implantation in a patient with complex regional pain syndrome
}

This article was published in the following Dove Press journal: Journal of Pain Research

\author{
Jaeyoung Yang \\ Yusun Choi \\ Juyoung Park \\ Junhyug Jeong \\ Bousung Lee \\ Kwanghaeng Lee \\ Jaedo Lee \\ Rakmin Choi
}

Department of Anesthesiology and Pain Medicine, Veterans Health Service Medical Center, Seoul, South Korea

Correspondence: Rakmin Choi Department of Anesthesiology and Pain Medicine, Veterans Health Service Medical Center, 53, Jinhwangdo-ro 6I-gil, Gangdong-gu, Seoul 05368, South Korea Tel +82 $22225 \quad 1473$

Email maxian0I@naver.com

\begin{abstract}
We successfully performed cervical spinal cord stimulator (SCS) surgery in a 27-year-old man with complex regional pain syndrome to control intractable pain. The SCS trial was performed twice to adjust the SCS coverage region. After permanent implantation surgery, the patient developed Horner's syndrome when the region near the C4 spinal dorsal root was stimulated. However, Horner's syndrome disappeared after changing the stimulation leads. This case report suggests that cervical SCS can be associated with superior cervical ganglion stimulation.
\end{abstract}

Keywords: spinal cord stimulator, Horner's syndrome, complex regional pain syndrome, superior cervical ganglion block

\section{Introduction}

Complex regional pain syndrome (CRPS) is a rare neuropathic condition characterized by spontaneous or evoked pain with allodynia, hyperalgesia, skin color change, edema, and altered hair or nail growth. ${ }^{1}$ A variety of pharmacological, psychological, and interventional treatment options are suggested to manage CRPS, but there is weak evidence supporting the effectiveness of pharmacological therapy. ${ }^{2}$ Multiple reports have suggested that spinal cord stimulator (SCS) is effective in managing CRPS. ${ }^{3,4}$ We report here a case of Horner's syndrome following cervical SCS implantation that resolved after stimulation lead change.

\section{Case report}

A 27-year-old man underwent surgical intervention for right radial styloid fracture 6 years ago. Several months after the operation, the patient complained of intense and severe burning pain (visual analog scale: 8/10), edema, reduced strength, cold allodynia, and hyperalgesia in the right forearm. He was diagnosed with CRPS type I by a pain management specialist. He underwent a series of right stellate ganglion blocks, which provided minimal pain relief only for the duration of the local anesthetics. The patient was given various medications such as carbamazepine, pregabalin, gabapentin, oxycodone, and fentanyl to manage the pain, but none of medicines provided complete pain relief. Furthermore, treatment by a physical therapist and pain psychologist was also not effective. After struggling with CRPS for 6 years, he was referred to our pain management clinic for SCS insertion. This case report was approved by the Veterans Health Service medical center institutional review board (ID: 2018-09-014). The patient 
provided written informed consent to have his case details and accompanying images to be published.

The results of preoperative laboratory tests, chest radiography, and electrocardiogram were normal. A percutaneous lead trial was performed with a Vectris lead (8-lead array, Medtronics, Minneapolis, MN, USA). The electrode lead was inserted into the cervical epidural space by using the loss of resistance technique. The upper tip of the electrode was positioned to reach the top of the $\mathrm{C} 3$ body under continuous fluoroscopy. The lead of spinal neurostimulator was located at the midline of the C3 body (Figure 1). In the testing of electrodes, complete coverage for the right arm was achieved with the stimulation of the second and third lead. After completing the first stimulation trial, the patient's pain intensity improved from 8 to 4 on the visual analog scale. In addition to the pain relief, he could touch cold water without allodynia.

On postoperative day (POD) 1 , his right arm pain aggravated and paresthesia from spinal cord stimulation was now experienced in the left instead of right arm. We tried stimulation of various electrodes to relieve the patient of his left-arm paresthesia, but to no avail. On cervical radiography, it was difficult to find the change in the lead location.

At POD 3, a second SCS trial was planned to relocate the lead. The permanent SCS lead was located to the right side of the $\mathrm{C} 3$ body to ensure right arm stimulation. The permanent lead was connected to an external pulse generator using an extension cable. After the second stimulation trial, the paresthesia from spinal cord stimulation was only achieved at the right arm of the patient from the stimulation of the second and third lead. At POD 4, he complained of minor inability to focus on nearby objects, but there was no specific problem observed in any physical and neurological examination. Further evaluation for myopia was postponed after SCS implantation. The effectiveness for pain relief between the first and second trial was similar.

On POD 6, a surgery for permanent implantation was performed under local anesthesia (Figures 2 and 3). The extension cable was removed, and a permanent lead was connected with implantable pulse generator (IPG, RestoreSensor SureScan MRI, Medtronics) buried under the skin of the right subclavicular area.

On POD 7, the patient developed right-sided meiosis and ptosis (Figure 4). There was no other neurological abnormality such as mental change, headache, motor weakness, or sensory abnormality. He was hemodynamically stable. His pupil size was $3 \mathrm{~mm}$ (right) and $5 \mathrm{~mm}$ (left) with normal light reflexes. He was diagnosed with Horner's syndrome based on his clinical examination findings.

Based on the location of the stimulation lead (C3), the stimulation of superior cervical ganglion was suspected to be the cause of Horner's syndrome. The stimulation lead was moved from the second and third to the third and fourth leads, respectively (Figure 3). After stimulation lead change, Horner's syndrome disappeared in 24 hours (Figure 5). No further invasive evaluation was required. His final SCS program was 0.5-1 $\mathrm{V}$ for voltage, $130 \mathrm{~Hz}$ for frequency, and $330 \mu$ s for pulse width.

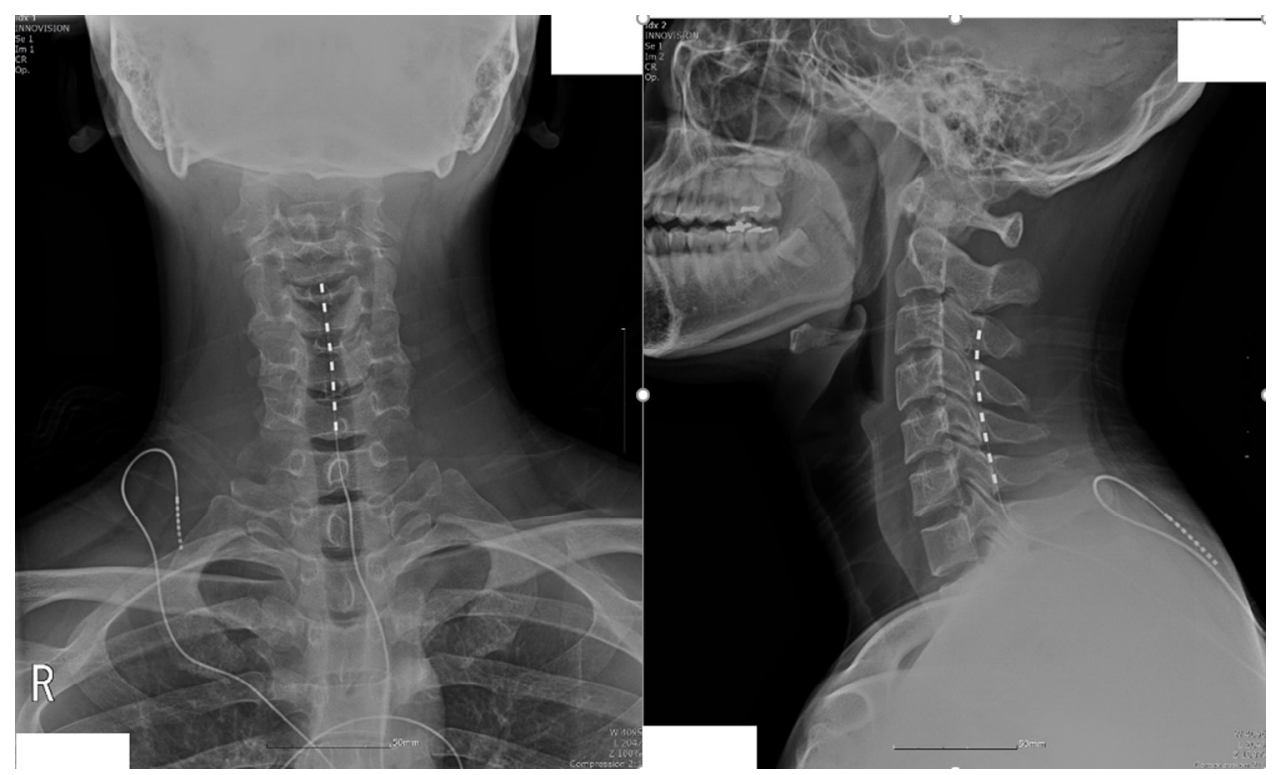

Figure I The cervical anteroposterior and lateral radiographs after the first spinal cord stimulator trial. 


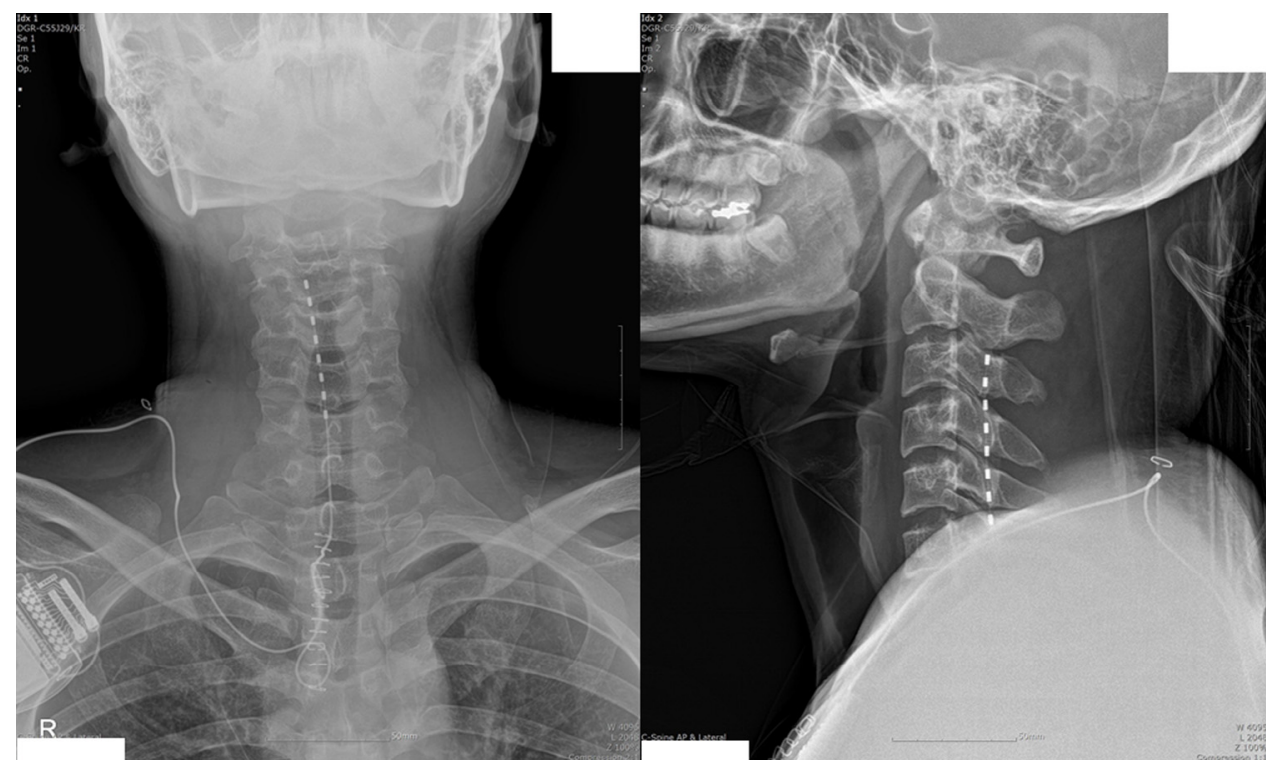

Figure 2 The cervical anteroposterior and lateral radiographs after permanent spinal cord stimulator implantation.

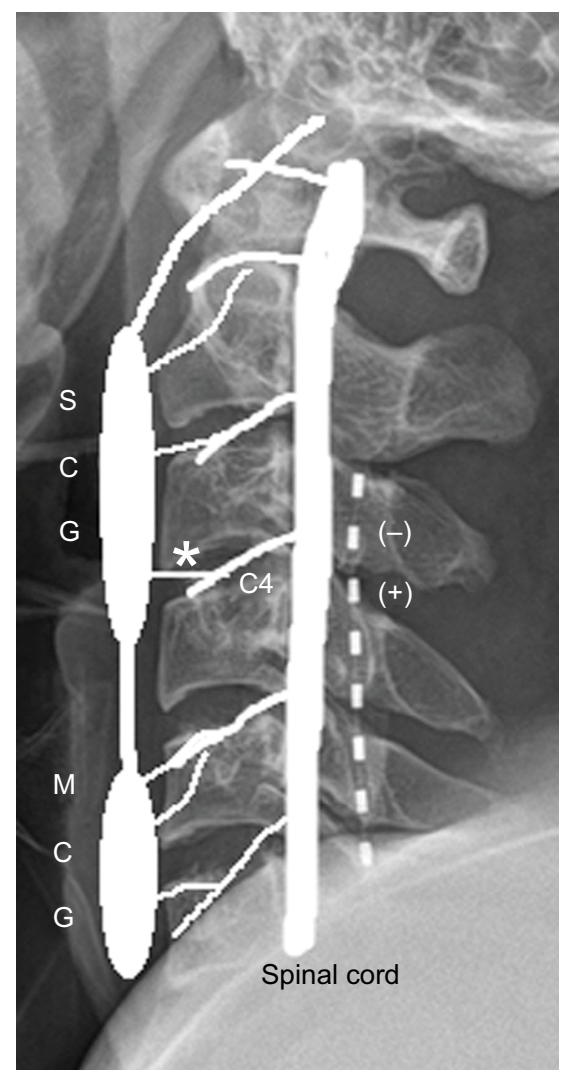

Figure 3 The schematic diagram of relevant anatomical structures and spinal cord stimulator.

Notes: The SCG is connected with C4 spinal nerve through the lateral branch of the SCG $(*)$. Active electrode is radiopaque and white on the radiograph. After the second trial, electrical stimulation was applied to the second (negative) and third (positive) electrodes.

Abbreviations: MCG, middle cervical ganglion; SCG, superior cervical ganglion.

\section{Discussion}

Intractable pain control with neuromodulation is one of the most rapidly developing medical fields. SCS has been used to treat chronic pain conditions such as failed back surgery syndrome and CRPS. ${ }^{5}$ Neuromodulation has minimal and reversible side effects. Although severe complications are rare, the incidence of minor complications is around $30 \%-40 \%{ }^{6}$

SCS complications are divided into two categories mechanical and biological. The incidence of mechanical complications $(24 \%-50 \%)$ is higher than that of biological complications (7.5\%). ${ }^{7}$ Mechanical complications include lead migration, lead fracture, and IPG failure. Biological complications include infection, IPG seroma, epidural hematoma, dural puncture, and neurological injury. ${ }^{6}$

To the best of our knowledge, this is the first report to describe that cervical SCS can be associated with reversible Horner's syndrome in a CRPS patient.

Horner's syndrome can result from the lesion affecting the oculosympathetic pathway. ${ }^{8}$ The anesthetic or analgesic procedures affecting the oculosympathetic pathway, namely the stellate ganglion blocks, interscalene block, brachial plexus, or thoracic epidural analgesia, could cause transient Horner's syndrome. ${ }^{7}$

Horner's syndrome is typically diagnosed with a triad of ipsilateral signs - ptosis, meiosis, and anhidrosis. However, anhidrosis could be absent or partial if the postganglionic fibers of the oculosympathetic pathway are disrupted. ${ }^{8}$ 


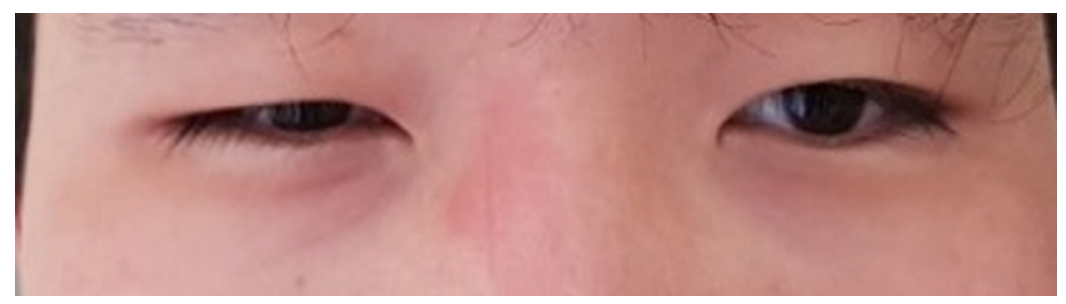

Figure 4 Eye photograph of the patient taken at postoperative day 7, after developing Horner's syndrome.

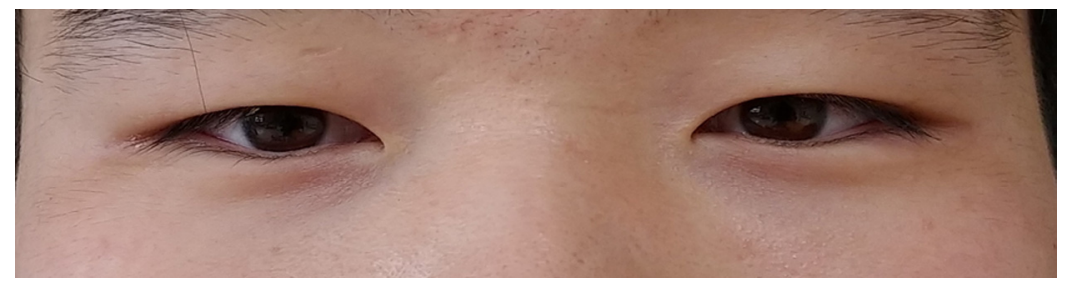

Figure 5 Eye photograph of the patient taken at postoperative day 8 after the change of stimulation lead; Horner's syndrome was resolved.

The oculosympathetic pathway consists of three parts: central (the first-order neuron), preganglionic (the secondorder neuron), and postganglionic (the third-order neuron). The first-order neuron (central fiber) travels from the posterolateral hypothalamus to the ciliospinal center at the C8-T2 level. The second-order neuron (preganglionic fiber) ascends the cervical sympathetic chain to synapse in the superior cervical ganglion. The third-order neuron (postganglionic fiber) emerges from the superior cervical ganglion and travels into the orbit with the trigeminal nerve. ${ }^{8}$

In this case report, Horner's syndrome developed after the lateral side of the $\mathrm{C} 4$ spinal dorsal root was stimulated. However, the initial stimulation of midline $\mathrm{C} 4$ did not cause Horner's syndrome.

Based on the lateral cervical radiography of the patient, the second and third stimulation leads were closely located to $\mathrm{C} 3$ vertebra, just next to the superior cervical ganglion. It did not seem likely that electrical current penetrated the cervical vertebrae and directly stimulated the superior cervical ganglion. In the rat model, Li et al reported that SCS can only be effective in a $2-8 \mathrm{~mm}$ range at $0.5-5 \mathrm{~mA}$ stimulation. ${ }^{9}$

In this case, the second and third leads were the negative and positive electrodes, respectively (Figure 3 ). The electrical current was injected into the second lead and flowed down to the third electrode. The outside of the neuron is more positively charged than the inside. When negative current was injected into the outside of the neuron, the cell membrane becomes depolarized and excited.
The possible mechanisms of neuromodulation in SCS could be divided into two groups: (1) supraspinal effect and (2) nonsupraspinal effect (local effect, peripheral, and segmental spinal cord mechanism of SCS). ${ }^{10}$

In nonsupraspinal effect (local effect), pain modulation can be achieved by the direct inhibition of small diameter sensory neurons and the activation of inhibitory interneuron at the dorsal column of the spinal cord. In the supraspinal effect, dorsal column stimulation can regulate descending pain inhibitory pathways such as the corticospinal track. ${ }^{10}$

In this case, both mechanisms could be involved in reversible Horner's syndrome. First, nonsupraspinal effect (local effect) of the SCS, mediated by the communicating nerve between the superior cervical ganglion and the cervical spinal nerve, could be the possible mechanism for transient (or reversible) Horner's syndrome.

Although the superior cervical ganglion receives main input from the ciliospinal center located at the C8-T2 level, the lateral branches of the superior cervical ganglion can communicate with the upper four cervical nerves (diagram of cervical sympathetic system: https://www.bartleby. com/107/216.html\#i844). ${ }^{11}$

Second, the ciliospinal center located at the C8-T2 level could be affected by the supraspinal effect of SCS. The ciliospinal center could be blocked by the dorsal column stimulation of the $\mathrm{C} 4$ level, thereby affecting the descending pain inhibitory pathway. 
The limitation of this case report is that it is unclear whether this kind of superior cervical ganglion stimulation is possible in the general population.

In conventional cervical SCS implantation, most cervical electrodes are located at the midline of the spinal cord to avoid stimulation of anterior spinal root, carrying motor signals. We believe this is why most cervical SCS implantations do not cause Horner's syndrome. In this case, the lateral branch of the superior cervical ganglion, communicating with the C4 spinal nerve, might be accidentally stimulated with the right-side displaced SCS electrode.

Superior cervical ganglion block is not so popular in clinical practice. However, it has been reported that superior cervical ganglion block could be effective in refractory facial and head pain. ${ }^{12,13}$ This report suggests that if a patient suffering from multiple pain regions involving the head and arms plans to undergo SCS implantation, the lateral placement of cervical electrode may help to improve the pain modulation.

In conclusion, if Horner's syndrome is observed after cervical SCS implantation, a positional change of stimulation leads should be the first intervention. Careful neurological and ophthalmological examinations should be performed to exclude other possible complications. After excluding all other possible causes, physicians should provide reassurance and comfort to those affected by Horner's syndrome.

\section{Disclosure}

The authors report no conflicts of interest in this work.

\section{References}

1. Bruehl S. Complex regional pain syndrome. BMJ. 2015;351:h2730.

2. Cossins L, Okell RW, Cameron H, Simpson B, Poole HM, Goebel A. Treatment of complex regional pain syndrome in adults: a systematic review of randomized controlled trials published from June 2000 to February 2012. Eur J Pain. 2013;17(2):158-173.

3. Forouzanfar T, Kemler MA, Weber WE, Kessels AG, van Kleef M. Spinal cord stimulation in complex regional pain syndrome: cervical and lumbar devices are comparably effective. $\mathrm{Br} J$ Anaesth. 2004;92(3):348-353.

4. Poree L, Krames E, Pope J, Deer TR, Levy R, Schultz L. Spinal cord stimulation as treatment for complex regional pain syndrome should be considered earlier than last resort therapy. Neuromodulation. 2013;16(2):125-141.

5. Song JJ, Popescu A, Bell RL. Present and potential use of spinal cord stimulation to control chronic pain. Pain Physician. 2014;17(3):235-246.

6. Verrills P, Sinclair C, Barnard A. A review of spinal cord stimulation systems for chronic pain. J Pain Res. 2016;9:481-492.

7. Deer TR, Mekhail N, Provenzano D, et al. The appropriate use of neurostimulation: avoidance and treatment of complications of neurostimulation therapies for the treatment of chronic pain. Neuromodulation Appropriateness Consensus Committee. Neuromodulation. 2014;17(6):571-597.

8. Cowie S, Gunn L, Madhavan P. Horner's syndrome secondary to epidural anaesthesia following posterior instrumented scoliosis correction. Asian Spine J. 2015;9(1):121-126.

9. Li J, Kong X, Gozani SN, Shi R, Borgens RB. Current-distance relationships for peripheral nerve stimulation localization. Anesth Analg 2011;112(1):236-241

10. Sivanesan E, Maher DP, Raja SN, Linderoth B, Guan Y. Supraspinal mechanisms of spinal cord stimulation for modulation of pain: five decades of research and prospects for the future. Anesthesiology. 2018.

11. Gray H. The cervical portion of the sympathetic system. Vol 7b. In: Anatomy of the Human Body. Philadelphia: Lea \& Febiger, 1918.

12. Elsner F, Radbruch L, Gaertner J, Straub U, Sabatowski R. Efficacy of opioid analgesia at the superior cervical ganglion in neuropathic head and facial pain. Schmerz. 2006;20(4):268-272.

13. Spacek A, Böhm D, Kress HG. Ganglionic local opioid analgesia for refractory trigeminal neuralgia. Lancet. 1997;349(9064):1521.
Journal of Pain Research

\section{Publish your work in this journal}

The Journal of Pain Research is an international, peer reviewed, open access, online journal that welcomes laboratory and clinical findings in the fields of pain research and the prevention and management of pain. Original research, reviews, symposium reports, hypothesis formation and commentaries are all considered for publication

\section{Dovepress}

The manuscript management system is completely online and includes a very quick and fair peer-review system, which is all easy to use. Visit http://www.dovepress.com/testimonials.php to read real quotes from published authors. 